S. Saint

A. M. Fendrick

\title{
Economic immunity in the ICU: continued support or pull the plug?
}

\author{
Accepted: 18 January 1999 \\ S. Saint $(\bowtie) \cdot$ A. M. Fendrick \\ Division of General Medicine, \\ Department of Internal Medicine, \\ University of Michigan Health System, Ann Arbor, \\ MI 48109, USA \\ S. Saint · A. M. Fendrick \\ Consortium for Health Outcomes, \\ Innovation, and Cost-Effectiveness Studies (CHOICES), \\ Department of Internal Medicine, \\ University of Michigan Health System, Ann Arbor, \\ MI 48109, USA \\ Mailing address: \\ 3116P Taubman Center, \\ 1500 E. Medical Center Drive, \\ University of Michigan Health System, Ann Arbor, \\ MI 48109, USA \\ e-mail: saint@umich.edu \\ Tel. + 1-(734)-936-5225 \\ Fax + 1-(734)-936-8944
}

Critically ill patients continue to be cared for in specialized (and costly) intensive care units (ICUs). Some have argued that the ICU symbolizes the fundamental dilemma of modern heath care - miracles occur but only at great expense [1]. The ICU has been protected from the explicit consideration of costs - perhaps due to our difficulty in accepting the explicit trade-offs between healthcare expenditures and life and death. Ironically, we believe the ICU is one of the most potent venues for economic analyses and that "immunity" from economic scrutiny is unwarranted for several reasons. First, methods to measure resource use in ICUs tend to be more sophisticated than in other segments of health care delivery, since the ICU frequently functions as an autonomous entity [2]. Second, randomized trials assessing the effectiveness of interventions involving the critically ill are common, as are severity-of-illness and risk-adjustment models developed exclusively for the critically ill, which allow for reliable clinical effectiveness estimates. Finally, relative to the incremental clinical benefits achieved by many ICU interventions, the economic costs of ICU care are substantial.

Indeed, the cost of intensive care has been estimated to consume $\$ 100$ billion per year in the United States [3]. Though ICUs comprise less than $10 \%$ of all hospital beds in the United States, they consume about $20 \%$ of all hospital costs [4]. This disproportionate amount of resources spent is not unique to the United States. The daily cost of intensive care management in the United Kingdom is $2-5$ times greater than that for general ward treatment [5]. Thus, rigorous economic evaluations of interventions in the ICU are necessary and welcome.

Economic evaluations have become increasingly important as a method to quantify trade-offs between improving clinical outcomes and increased health care expenditures in an era of escalating health care costs [6]. As budgets tighten, economic analyses can help decision-makers set priorities for funding health care programs by allowing a comparative quantitative framework [7]. Specifically, these analyses aid decision-makers in choosing among several different interventions based on the costs and outcomes of each intervention. Scarce resources can then be expended on those interventions yielding the greatest clinical benefit relative to the extra cost (i. e., cost-effectiveness ratio) or, in other words, providing the "most juice for the least amount of squeeze."

Accordingly, we appreciate the contribution of Combier and colleagues [8] for their economic analysis of terlipressin-glycerin trinitrate (TER-GTN) in the management of acute gastrointestinal bleeding in cirrhotic patients with ruptured esophageal varices (ROV). Their finding that the incremental cost of a death avoided associated with this innovation was approximately FF 
25,000 (or about $\$ 4500$ ) makes this intervention worth strong consideration provided: 1) the effectiveness estimate is reasonable and reproducible, 2) the cost estimates are appropriate and accurate, 3 ) sensitivity analyses reveal that the clinical and economic estimates used are robust, and 4) the results of this study can be compared to other appropriate management strategies for this clinical scenario.

\section{Are the effectiveness estimates reasonable?}

Economic evaluations of health care interventions depend upon solid clinical evidence of effectiveness in order to establish benefits and risks. The validity of the clinical data is crucial to the overall usefulness of the analysis. Many economic evaluations rely on a single randomized trial or a single observational study to estimate clinical benefit. Some studies rely on less rigorous or non-scientific sources of information for outcome assessment, such as clinical opinions and expert panels. Estimates derived from large-scale, multi-center trials are widely considered the "gold standard," but these data are often not available. Of concern to researchers and policy analysts is the accuracy and precision of the estimated intervention benefit derived from a single study. This is a particularly relevant point for those who rely on deterministic decision analytic models for cost-effectiveness predictions where point estimates are commonly used.

Ideally, one would want several studies of the same interventions (treatment and comparator) within a similar population from which to determine an average benefit. A few economic evaluations have indeed used meta-analysis to estimate intervention effects [9]. However, interventions frequently have not been evaluated in several separate trials, thus, meta-analysis can not be performed. How good then is an estimate of effectiveness derived from a single study? Provided that the randomized trial upon which this study is based was rigorous and free of obvious bias, the economic evaluation is appropriate.

\section{Are the cost estimates appropriate and accurate?}

Before deciding what costs to include in an economic analysis, the perspective of the evaluation must be explicitly stated at the outset. Many experts have recommended that the societal perspective be assumed [10]. The societal perspective is particularly important if comparability between different economic analyses is desired. Because the perspective of the hospital is used in the study by Combier et al., the investigators use a more restrictive economic viewpoint. We believe that the most appropriate perspective to be taken when per- forming an economic analysis is that of the decisionmaker. If a hospital is the primary decision-maker regarding the adoption of a new intervention, then a narrower economic perspective can be justified. However, the potential bias assumed by taking a non-societal perspective should be considered and discussed. If Combier and colleagues had taken the societal perspective, and thus included such costs as time lost from work for both patients and visitors, the intervention would probably have been even more cost-effective.

The external validity of the cost estimates should also be examined. Regional variability in market forces often lead to substantial variability in cost estimates in different health systems, even for similar services. Thus, even if we assume that the cost estimates used by Combier et al. are appropriate and accurate, the expenses incurred in France may be an inaccurate estimate of the economic costs associated with this intervention in other countries. In short, despite the fact that a clinical benefit of an intervention could be demonstrated in every clinical setting, the cost-effectiveness ratios would vary considerably depending on the local economic venue. Thus, an intervention that may appear "cost-effective" in one country (e.g., with a cost-effectiveness ratio less than $\$ 50,000$ per life year saved), the same intervention may be considered too expensive in other countries.

\section{Do sensitivity analyses adequately assess the robustness of the clinical and economic estimates?}

Sensitivity analyses assess how much the uncertainty in the estimates of effect or cost will impact the overall results. In order to assess the robustness of effectiveness estimates, the upper and lower values of the $95 \%$ confidence interval of the difference between the intervention and comparator groups should be used in a oneway sensitivity analysis. Cost estimates should also be varied across a reasonable range of estimates. If the cost-effectiveness ratio remains reasonable (e.g., less than $\$ 50,000$ per life year saved) over the entire range of values, the conclusions of the analysis are strengthened and the concerns over the parameters used are lessened. Unfortunately, Combier and colleagues did a much more limited evaluation of the uncertainty of their analysis than what we routinely recommend; thus, the uncertainty of the parameters chosen (for both effectiveness and costs) cannot be adequately assessed.

\section{Comparability to other interventions}

The clinical effect of the intervention evaluated in the study by Combier and colleagues, as demonstrated by the short-term mortality reduction, is indeed impressive. We are unaware of other interventions for ROV 
in cirrhotic patients that have been found to be as clinically and economically worthwhile. Thus, it is quite likely that the outcome evaluated - cost per death avoided is cost-effective. However, since patients with cirrhosis who have an acute upper gastrointestinal bleed and survive have a high short-term mortality, it is necessary that more information on the duration of survival (and preferably the quality of life after survival) be provided in order to compare TER-GTN with other interventions that may have a less dramatic short-term, but longer lasting, effect. Though the authors do comment on this point in their discussion, it is unfortunate that the cost per death avoided end point could not be expressed as a cost per life year saved or cost per quality-adjusted life year saved (QALY) in order to allow comparison to different health care interventions. These two end points have been recommended in most, if not all, guidelines for reporting cost-effectiveness analyses. If most survivors died soon after the administration of this innovative product (e.g., after 60 days), then the cost-effectiveness ratio reported by the authors would appear better than the actual cost-effectiveness ratio of
TER-GTN as measured in terms of years of life saved or QALY. Thus, though TER-GTN does appear cost-effective in improving 42-day mortality in cirrhotic patients experiencing an upper gastrointestinal tract bleed, additional data on the long-term survival of these patients are necessary to determine whether the benefits remain great enough to recommend the widespread use of this therapy.

In conclusion, we applaud Combier and colleagues for their important contribution to the literature. We hope that well-done economic evaluations become even more common in the ICU. Though several important meta-analyses and cost-effectiveness analyses of interventions in the critically ill have recently been published [11-13], additional work is needed if the ICU is to remain an economically viable "miracle producer." Several excellent guidelines have been published for both producers and users of economic evaluations [10, $14,15]$. Given the heightened concern about healthcare costs around the globe, now is the time to overcome the immunity that the ICU has enjoyed from economic scrutiny.

\section{References}

1. Bone RC (1995) Economic analysis of the intensive care unit: a dilemma [editorial; comment]. Crit Care Med 23(5):805

2. Multz AS, Chalfin DB, Samson IM et al. (1998) A "closed" medical intensive care unit (MICU) improves resource utilization when compared with an "open" MICU. Am J Respir Crit Care Med 157(5 Pt 1):1468-1473

3. Raffin TA (1989) Intensive care unit survival of patients with systemic illness. Am Rev Respir Dis 140(2 Pt 2):S28-35

4. Kirton OC, Civetta JM, Hudson Civetta J (1996) Cost effectiveness in the intensive care unit. Surg Clin North Am 76(1):175-200

5. Ridley S, Biggam M, Stone P (1993) A cost-benefit analysis of intensive therapy. Anaesthesia 48(1):14-19

6. Chalfin DB, Cohen IL, Lambrinos J (1995) The economics and cost-effectiveness of critical care medicine. Intensive Care Med 21(11):952-961
7. Detsky AS, Naglie IG (1990) A clinician's guide to cost-effectiveness analysis [see comments]. Ann Intern Med 113(2):147-154

8. Combier E, Levacher S, Letoumelin P, Joseph A, Pourriat JL, De Pouvourville G (1999) Cost-effectiveness analysis of the terlipressin-glycerin trinitrate combination in the pre-hospital management of acute gastrointestinal haemorrhage in cirrhotic patients. Intensive Care Med 25: 364-370

9. Saint S, Veenstra DL, Sullivan SD (1999) The use of meta-analysis in costeffectiveness analysis: issues and recommendations. Pharmacoeconomics 15: $1-8$

10. Gold MR, Siegel JE, Russell LB, Weinstein MC (1996) Cost-effectiveness in health and medicine. Oxford University Press, New York

11. Veenstra DL, Saint S, Saha S, Lumley T, Sullivan SD (1999) Efficacy of antiseptic-impregnated central venous catheters in preventing catheter-related bloodstream infection: a meta-analysis. JAMA 281: 261-267
12. Cook DJ, Reeve BK, Guyatt GH et al. (1996) Stress ulcer prophylaxis in critically ill patients. Resolving discordant meta-analyses. JAMA 275(4):308-314

13. Ben Menachem T, McCarthy BD, Fogel R et al. (1996) Prophylaxis for stress-related gastrointestinal hemorrhage: a cost effectiveness analysis. Crit Care Med 24(2):338-345

14. Anonymous (1984) How to read clinical journals: VII. To understand an economic evaluation (part A). Can Med Assoc J 130(11):1428-1434

15. Anonymous (1984) How to read clinical journals: VII. To understand an economic evaluation (part B). Can Med Assoc J 130(12):1542-1549 